\title{
Extended VIKOR method with fuzzy cross-entropy of interval-valued intuitionistic fuzzy sets
}

\author{
Xinye Zhao, Teng'an Zou, Shanliang Yang, Mei Yang \\ Mechatronics and Automation School \\ National University of Defense Technology \\ Changsha, China \\ zhaoxinye@nudt.edu.cn
}

\begin{abstract}
On the foundation of employing the new induced generalized intuitionistic fuzzy ordered weighted averaging operator to aggregate all decision matrices into a collective decision matrix, for the two special situations where the information on attribute weights is incomplete and inconsistent or completely unknown, the feasibility of utilizing linear optimization technology as a core element within the accuracy function to obtain criterion weight is being investigated, and a novel model of entropy weights for determining the exact criterion weight is established, respectively. In this paper, the extended VIKOR method is developed to solve group decision making problems assuming that compromising is acceptable to resolve conflicts.
\end{abstract}

Keywords-interval-valued intuitionistic fuzzy sets (IVIFS); entropy, group multiple criteria decision making (GMCDM); cross entropy; VIKOR

\section{INTRODUCTION}

In 1986, Atanassov [1] proposed the concepts of intuitionistic fuzzy sets(IFS) where each element in an intuitionistic fuzzy set has a membership degree and a non-membership degree between $[0,1]$ respectively. Atanassov and Gargov (1989) [2] introduced the interval-valued intuitionistic fuzzy set (IVIFS) as a further generalization of IFS. For their advantage of coping with more imprecise information, many authors have applied the IVIFS theories to deal with group multiple criteria decision making (GMCDM) problems.

Recently, the VIKOR method has been greatly undertaken by researchers to address decision making problems, particularly in the field of alternative selection with more accurate solution [3]. This paper extends the VIKOR method to resolve the problem of evaluation and ranking the potential alternatives based on cross-entropy concept.

\section{GROUP DECISION MAKING PROBLEM WITH INTERVAL-VALUED INTUITIONISTIC FUZZY DATA}

In this section, we proceed to the cross-entropy method to solve GMCDM problems in which all preference information provided by DMs is expressed as interval-valued intuitionistic fuzzy decision matrices where each of the elements is characterized by IVIFS, and the information about attribute weights is partially known or completely unknown.

\section{A. Problem formulation}

For the sake of convenience, we describe the GMCDM problem by means of following sets:

1) A set of $m$ alternatives called $A_{i}(i=1,2, \ldots, m)$;

2) A set of $n$ decision criteria called $c_{j}(j=1,2, \ldots, n)$;

3) A set of $n$ weighting vector of decision criteria called $w_{j}(j=1,2, \ldots, n)$;

4) A set of $k$ DMs called $D_{k}(k=1,2, \ldots, t)$;

5) A set of $k$ decision matrix called $R_{k}(k=1,2, \ldots, t)$;

6) A set of $k$ weighting vector of DMs called $u_{k}(k=$ $1,2, \ldots, t)$;

where $R_{k}$ can be represented as follows:

$$
\begin{array}{r}
R_{k}=\left(\widetilde{r}_{i j}\right)_{m \times n}=\left(\left[\mu_{i j}^{l k}, \mu_{i j}^{u k}\right],\left[\nu_{i j}^{l k}, \nu_{i j}^{u k}\right]\right)= \\
{\left[\begin{array}{ccc}
\left(\left[\mu_{11}^{l k}, \mu_{11}^{u k}\right],\left[\nu_{11}^{l k}, \nu_{11}^{u k}\right]\right) & \ldots & \left(\left[\mu_{1 n}^{l k}, \mu_{1 n}^{u k}\right],\left[\nu_{1 n}^{l k}, \nu_{1 n}^{u k}\right]\right) \\
\left(\left[\mu_{21}^{l k}, \mu_{21}^{u k}\right],\left[\nu_{21}^{l k}, \nu_{21}^{u k}\right]\right) & \ldots & \left(\left[\mu_{2 n}^{l k}, \mu_{2 n}^{u k}\right],\left[\nu_{2 n}^{l k}, \nu_{2 n}^{u k}\right]\right) \\
\vdots & \vdots & \ddots \\
\left(\left[\mu_{m 1}^{l k}, \mu_{m 1}^{u k}\right],\left[\nu_{m 1}^{l k}, \nu_{m 1}^{u k}\right]\right) & \ldots & \left(\left[\mu_{m n}^{l k}, \mu_{m n}^{u k}\right],\left[\nu_{m n}^{l k}, \nu_{m n}^{u k}\right]\right)
\end{array}\right]}
\end{array}
$$

\section{B. Attribute weights are partially known}

In reality, there are often cases that the DM only knows partial weight information, that means, available information about weights is not sufficient for exact definition of membership and non-membership degrees for certain elements. Under this situation, one is only sure that the weight vector $w_{j}$ satisfy $w_{j} \geq 0$ and $\sum_{j=1}^{n} w_{j}=1$. As a rule, the incomplete attribute weight information can be expressed as follows, for $i \neq j$ :

1) A weak ranking: $\left\{w_{i} \geq w_{j}\right\}$;

2) A strict ranking: $\left\{w_{i}-w_{j} \geq \delta(\delta>0)\right\}$;

3) A ranking with multiples: $\left\{w_{i} \geq \alpha_{i} w_{j}\left(\alpha_{i} \in[0,1]\right)\right\}$;

4) An interval form: $\left\{\delta_{i} \leq w_{i} \leq \delta_{i}+\varepsilon_{i}\left(0 \leq \delta_{i}<\right.\right.$ $\left.\left.\delta_{i}+\varepsilon_{i} \leq 1\right)\right\}$

5) A ranking of differences: $\left\{w_{i}+w_{j} \geq w_{k}+w_{l}(i \neq\right.$ $j \neq k \neq l)\}$;

A reasonable weight vector should make the total accuracy function values of the alternatives as large as possible. So an interesting and important issue is how to obtain 
the principle results in the following multiple objective optimization model for determining the weight vector. In this generalization, we suggest a linear-programming procedure based on a accuracy function value maximization problem such that the overall function values of entire alternatives depend upon their associated weighting vector variables.

\section{Procedure 1:}

Step 1: Calculate the overall accuracy function values of each alternative $A_{i}(i=1,2, \ldots, m)$ by the accuracy function:

$$
\begin{gathered}
s(X)=\frac{a+b-d(1-b)+c(1-a)}{2} \\
s\left(A_{i}\right)=\sum_{j=1}^{n} w_{j} s\left(r_{i j}\right) \quad i=1,2, \ldots, m .
\end{gathered}
$$

Step 2: Clearly, the greater the value $s\left(A_{i}\right)$, the better the alternative $A_{i}$. If we consider the alternative $A_{i}$ only, then a corresponding vector of attribute weights $w^{(i)}=\left(w_{1}, w_{2}, \ldots, w_{n}\right)^{T}$ should be decided. Therefore, establish the following single-objective programming model to maximize $s\left(A_{i}\right)$ :

$$
\begin{aligned}
& \text { M1) Maximize: } \quad s\left(A_{i}\right)=\sum_{j=1}^{n} w_{j} s\left(r_{i j}\right) \\
& \text { s.t. }\left\{\begin{array}{l}
w^{(i)}=\left(w_{1}, w_{2}, \ldots, w_{n}\right)^{T} \in H \\
w_{j} \geq 0 \\
\sum_{j=1}^{n} w_{j}=1
\end{array}\right.
\end{aligned}
$$

Step 3: By solving the model M1, we obtain the optimal solution $w^{(i)}$ corresponding to the alternative $A_{i}$. After determining the weight vector $w_{j}$ respectively, in order to consider all the alternatives $A_{j}(j=1,2, \ldots, m)$ as a whole, we construct the total weight matrix $W=\left\{w_{j}^{(1)}, w_{j}^{(2)}, \ldots, w_{j}^{(m)}\right\}$ of the optimal solutions as follows:

$$
W=\left[\begin{array}{cccc}
w_{11}^{(1)} & w_{12}^{(1)} & \ldots & w_{1 n}^{(1)} \\
w_{21}^{(2)} & w_{22}^{(2)} & \ldots & w_{2 n}^{(2)} \\
\vdots & \vdots & \ddots & \vdots \\
w_{m 1}^{(m)} & w_{m 2}^{(m)} & \ldots & w_{m n}^{(m)}
\end{array}\right]
$$

Step 4: Then calculate the normalized eigenvector $\psi=\left(\psi_{1}, \psi_{2}, \ldots, \psi_{n}\right)$ of $\Psi=(S W)^{T}(S W)$. After that we construct a combined weight vector as follows:

$$
w=W \psi=\left[\begin{array}{cccc}
w_{11}^{(1)} & w_{12}^{(1)} & \ldots & w_{1 n}^{(1)} \\
w_{21}^{(2)} & w_{22}^{(2)} & \ldots & w_{2 n}^{(2)} \\
\vdots & \vdots & \ddots & \vdots \\
w_{m 1}^{(m)} & w_{m 2}^{(m)} & \ldots & w_{m n}^{(m)}
\end{array}\right]\left[\begin{array}{c}
\psi_{1} \\
\psi_{2} \\
\vdots \\
\psi_{n}
\end{array}\right]
$$

$$
\begin{aligned}
& \text { And we derive the weight vector } \\
& w=\left(w_{1}, w_{2}, \ldots, w_{n}\right)^{T} \text { of the criteria } \\
& c_{j}(j=1,2, \ldots, n) \text { finally. }
\end{aligned}
$$

\section{Attribute weights is completely unknown}

In the following, we introduce some basic concepts related to entropy and suggest a new method of calculating entropy measures for IVIFSs.

Definition 1: A real-valued function $E$ : IVIFS $(\mathrm{X}) \rightarrow[0,1]$ is called an entropy for IVIFSs, if it satisfies the following axiomatic requirements[4]:

1) $E(A)=0$ iff $\mathrm{A}$ is a crisp set;

2) $E(A)=1$ iff $\left[\mu_{A}^{L}(x), \mu_{A}^{U}(x)\right]=\left[\nu_{A}^{L}(x), \nu_{A}^{U}(x)\right]=$ $[0,0]$

3) $E(A)=E\left(A^{C}\right)$;

4) $E(A) \geq E(B)$, if $A \subseteq B$ when $\mu_{A}^{L} \leq \mu_{B}^{L}, \mu_{A}^{U} \leq \nu_{B}^{U}$, $\nu_{A}^{L} \leq \nu_{B}^{L}, \nu_{A}^{U} \leq \nu_{B}^{U}$

In the first place, we give an entropy measure for IFSs. For each $A \in \operatorname{IF} S(X), x \in X$, define $\widetilde{E}(A)$ by

$$
\widetilde{E}(A)=1-\left(\mu_{A}(x)+\nu_{A}(x)\right) \tan \frac{\pi}{4}\left(\mu_{A}(x)+\nu_{A}(x)\right)
$$

Furthermore, for any $A \in I V I F S s$, we propose the following entropy measure on A. For each $A \in \operatorname{IVIFS}(X)$, define $E(A)$ by

$$
\begin{aligned}
& E(A)=1-1-\left(\bar{\mu}_{A}(x)+\bar{\nu}_{A}(x)\right) \tan \frac{\pi}{4}\left(\bar{\mu}_{A}(x)+\bar{\nu}_{A}(x)\right) \\
& \text { where } \quad \bar{\mu}_{A}(x)=\mu_{A}^{L}(x)+\rho\left(\mu_{A}^{U}(x) \quad-\quad \mu_{A}^{L}(x)\right), \\
& \bar{\nu}_{A}(x)=\nu_{A}^{L}(x)+\rho\left(\nu_{A}^{U}(x)-\nu_{A}^{L}(x)\right), \rho \in(0,1) .
\end{aligned}
$$

The proof is omitted here.

For the special situations where the information about criterion weights $w_{j}$ is completely unknown, we develop a practical method based on entropy measures of IVIFSs to obtain the attribute weights as follows.

Procedure 2:

Step 1. Calculate the IFIVS entropy matrix $E_{m n}$ of decision matrix $R$ :

$$
E_{m n}=\left[\begin{array}{cccc}
E_{11} & E_{12} & \ldots & E_{1 n} \\
E_{21} & E_{22} & \ldots & E_{2 n} \\
\vdots & \vdots & \ddots & \vdots \\
E_{m 1} & E_{m 2} & \ldots & E_{m n}
\end{array}\right]
$$

where $E_{i j}=1-\left(\bar{\mu}_{i j}(x)+\bar{\nu}_{i j}(x)\right) \tan \frac{\pi}{4}\left(\bar{\mu}_{i j}(x)+\right.$ $\left.\bar{\nu}_{i j}(x)\right), \quad \bar{\mu}_{i j}(x)=\mu_{i j}^{L}(x)+\rho\left(\mu_{i j}^{U}(x)-\mu_{i j}^{L}(x)\right)$, $\bar{\nu}_{i j}(x)=\nu_{i j}^{L}(x)+\rho\left(\nu_{i j}^{U}(x)-\nu_{i j}^{L}(x)\right), \rho \in(0,1)$, $i=1,2, \ldots, m, j=1,2, \ldots, n$. 
Step 2. Utilize Eq.10 to transform $E_{m n}$ into the normalized intuitionistic fuzzy decision $\widehat{E}_{m n}$.

$$
\begin{gathered}
\widehat{E}_{i j}=\frac{E_{i j}}{\sum_{i=1}^{m} \sum_{j=1}^{n} E_{i j}} \\
\widehat{E}_{m n}=\left[\begin{array}{cccc}
\widehat{E}_{11} & \widehat{E}_{12} & \ldots & \widehat{E}_{1 n} \\
\widehat{E}_{21} & \widehat{E}_{22} & \ldots & \widehat{E}_{2 n} \\
\vdots & \vdots & \ddots & \vdots \\
\widehat{E}_{m 1} & \widehat{E}_{m 2} & \ldots & \widehat{E}_{m n}
\end{array}\right]
\end{gathered}
$$

Step 3. Establish an exact model of entropy weights for determining the criteria weight:

$$
w_{j}=\frac{1-\varphi_{j}}{n-\sum_{j=1}^{n} \varphi_{j}}
$$

where $w_{j} \in(0,1), \quad \sum_{j=1}^{n} w_{j}=1$, $\varphi_{j}=\frac{1}{m} \sum_{j=1}^{m} \widehat{E}_{T A}\left(A_{j}\right), \varphi_{j} \in[0,1]$.

According to the entropy theory, if the entropy value for each criterion is smaller across alternatives, it should provide DMs with more useful information. Consequently, the criterion should be assigned a bigger weight. Otherwise, such a criterion will be supposed unimportant by most DMs, and the criterion should be evaluated as a relatively small weight.

\section{VLSEKRITERIJUMSKA OPTIMIZACIJA I KOMPROMISNO RESENJE(VIKOR)}

The VIKOR method was developed by Opricovic and Tzeng (2002) [5]. Assuming that compromising is acceptable to resolve conflicts, VIKOR focuses on ranking and sorting a set of alternatives against various, or possibly conflicting and non-commensurable decision criteria. In the decision of strategy, the VIKOR method is more favorable than TOPSIS because the linear normalization does not depend on the evaluation unit of a criterion function, whereas the normalized values by vector normalization in the TOPSIS method may depend on the evaluation unit [6].

Definition 2: Development of the VIKOR method started with the following form of $L_{p}$-metric

$$
\begin{aligned}
l_{p, j}=\left\{\sum_{i=1}^{n}\left[w_{i}\left(f_{i}^{*}-f_{i j}\right) /\left(f_{i}^{*}-f_{i}^{-}\right)\right]^{p}\right\}^{1 / p}, \\
1 \leq p \leq \infty ; j=1,2, \ldots, J
\end{aligned}
$$

The measure $l_{p, j}$ was bringed forward by Duckstein and Opricovic [7] and it represents the distance of the alternative $A_{j}$ to the ideal solution. The compromise solution $F^{c}=\left(f_{1}^{c}, \ldots, f_{n}^{c}\right)$ is a feasible solution which is the 'closest' one to the ideal $F^{*}$. And compromise denotes an agreement arrived by mutual concessions, represented by $\triangle f_{i}=f_{i}^{*}-f_{i}^{c}(i=1, \ldots, n)$.
Definition 3: Let A,B be two IVIFSs, the differences between A and B can be expressed by cross-entropy between IVIFSs $\mathrm{A}$ and $\mathrm{B}$, which is defined as below.

$$
E_{\text {cross }}=\left|f_{A}-f_{B}\right|=S(A, B)
$$

where $S(A . B)=D(A, B)+D(B, A)$. For a definition of these functions, we refer the reader to [8].

The VIKOR calculation steps, of which there are five, are shown as follows:

Procedure 3:

Step 1: Decide the best $f_{i}^{*}$ and the worst $f_{i}^{c}$ values of all criterion functions $(i=1,2, \ldots, n)$. If the $i$ th function stands for a benefit, then $f_{i}^{*}=\max _{j} f_{i j}$ and $f_{i}^{-}=\min _{j} f_{i j}$;

Step 2: Calculate the values $S_{j}$ and $R_{j}$ by $S_{j}=\sum_{i=1}^{n} w_{i}\left(f_{i}^{*}-f_{i j}\right) /\left(f_{i}^{*}-f_{i}^{-}\right)$and $R_{j}=\max _{i}\left[w_{i}\left(f_{i}^{*}-f_{i j}\right) /\left(f_{i}^{*}-f_{i}^{-}\right)\right]$, where $w_{i}$ are the weights of criteria expressing their relative importance, given by former procedures;

Step 3: Calculate the values $Q_{j}(j=1,2, \ldots, J)$, by the relation $Q_{j}=v\left(S_{j}-S^{*}\right) /\left(S^{-}-S^{*}\right)+(1-$ $v)\left(R_{j}-R^{*}\right) /\left(R^{-}-R^{*}\right), \quad S^{*}=\min _{j} S_{j}$, $S^{-}=\max _{j} S_{j}, \quad R^{*}=\min _{j} R_{j}, \quad R^{-}=\max _{j} R_{j} . \quad v$ is introduced as the weight of the strategy of the maximum group utility; commonly assign $v=0.5$;

Step 4: Rank the alternatives, sorting by the values $S, R$ and $Q$, in decreasing order. The results are three ranking lists;

Step 5: Propose a compromise solution, the alternative $\left(a^{\prime}\right)$ which is ranked optimal by the measure $Q$ (minimum) if it satisfies the following two conditions:

Condition 1: Acceptable advantage: $Q\left(a^{\prime \prime}\right)-Q\left(a^{\prime}\right) \geq D Q$, where $a^{\prime \prime}$ is the alternative with second position in the ranking list given by $D Q=1 /(J-1), J$ is the number of alternatives.

Condition 2: Acceptable stability in decision-making: Alternative $a^{\prime}$ also has to be the best ranked by $S$ and/or $R$. This solution is stable in a decision-making process, which could be voting by 'majority rule' (when $v>0.5$ is needed) or 'by consensus' $(v \approx 0.5)$ or 'with veto' $(v<0.5)$.

When either of the conditions is not satisfied, then a set of compromise solutions are proposed, which consists of:

- Alternatives $a^{\prime}$ and $a^{\prime \prime}$ if only condition 2 is not satisfied, or;

- Alternatives $a^{\prime}, a^{\prime \prime}, \ldots, a^{(m)}$ if only condition $\mathbf{1}$ is not satisfied, and $a^{(m)}$ is determined by the relation $Q\left(a^{(m)}\right)-Q\left(a^{\prime}\right) \approx 1 /(J-1)$ for maximum $\mathrm{M}$. 
The characteristics of VIKOR are considering multiple practical problems, adopting multi-criteria solution to discuss the subjective cognition and objective appraisal. In connection with the linear normalization used in $S_{j}$ and $R_{j}$, the method makes an assumption that the DMs are willing to approve these trade-offs. The VIKOR method can be started without interactive participation of DMs, but the DMs may preside over approving the final solution and individual preference must be included.

\section{THE PROPOSED APPROACH FOR GMCDM}

Within this section, we are going to propose an extended version of VIKOR method that is developed to deal with a GMCDM problem. On the basic of the investigation before, now the proposed method is used to identify the best alternative.

Procedure 4:

Step 1: Establish group decision matrix $R_{k}$ by Eq.1, for each DM.

Step 2: Utilize the decision information given in matrix $R_{k}$, and the $\mathrm{I}-$ $\left.\operatorname{GIIFOWA}\left(\left\langle u_{1}, \widetilde{\alpha_{1}}\right\rangle,\left\langle u_{2}, \widetilde{\alpha_{2}}\right\rangle\right), \ldots,\left\langle u_{n}, \widetilde{\alpha_{n}}\right\rangle\right)$

operator [9] which has the associated weighting vector $w=\left(w_{1}, w_{2}, \ldots, w_{t}\right)^{T}$ to aggregate all the intuitionistic fuzzy decision matrices $R_{k}$ into a collective decision matrix $R$, where $u$ in $\langle u, \widetilde{\alpha}\rangle$ is $u_{k}$ (the weighting vector of DMs).

Step 3: According to two distinct occasions of criterion weight knownquantity, choose the pertinent solution.

- If the criteria weights are not known exactly, but their ranges are known, then set up and follow Procedure 1 to solve the linear programming problem (M1) for obtaining the weight vector.

- If the information about criterion weight $w_{j}$ is completely unknown, for determining the criterion weight $w_{j}$ from the decision matrix $D_{k}$, we can establish an exact model of entropy weights to obtain the information quantity by Procedure 2.

Step 4: Because exsits two disparate characteristics of criteria: revenue index and cost index. Reckon the best interval values of the criterion differently in succession, for revenue index: $c_{i}=\left(\left[\max \mu^{L}, \max \mu^{U}\right],\left[\min \nu^{L}, \min \nu^{U}\right]\right)$; for cost index: $c_{i}=$ ([min $\left.\left.\mu^{L}, \min \mu^{U}\right],\left[\max \nu^{L}, \max \nu^{U}\right]\right)$. Then according to the collective intuitionistic fuzzy information given in matrix R, follow Procedure 3 to derive the collective overall $S, R$ and $Q$ value of the alternative $A_{i}$ and rank them respectively, where $w_{j}$ is the weighting vector of the criterion.

\section{CONCLUSION}

VIKOR is a useful method in multi-criteria decision making, the obtained compromise solution could be accepted by the DMs because it provides a maximum group utility of the majority, and a minimum of the individual regret of the opponent. In this paper, we delve into GMCDM problem within IVIFS domain and introduce an extended VIKOR method of IVIFSs that differs from that of TOPSIS method used mostly, resolving the problem of evaluation and ranking the potential alternatives in detail.

This paper addresses a complex GMCDM problem with requirements spanning diverse areas of knowledge. Although the approach presented here is still in the research stage and thus is a work in progress, the ideas behind its implementation have the potential to provide insights to researchers and practitioners in the field of IVIFS and decide making. In addition, determining the sufficient and necessary conditions for the proposed method is still a problem that will be addressed in our future work.

\section{ACKNOWLEDGMENT}

This work is supported by Chinese National Natural Science Foundation (61074108).

\section{REFERENCES}

[1] K. Atanassov. Intuitionistic fuzzy sets. Fuzzy Sets and Systems, 20(1):87-96, 1986.

[2] K. Atanassov and G. Gargov. Interval valued intuitionistic fuzzy sets. Fuzzy Sets and Systems, 31(3):343-349, 1989.

[3] Ali Shemshadi, Hossein Shirazi, Mehran Toreihi, and M.J. Tarokh. A fuzzy vikor method for supplier selection based on entropy measure for objective weighting. Knowledge-Based Systems, 38:12160-12167, 2011.

[4] X.D. Liu and S.H. Zhang. Entropy and subsethood for general interval-valued intuitionistic fuzzy sets. L. Wang, Y. Jin (Eds.), FSKD 2005, LNAI 3613:42-52, 2005.

[5] S. Opricovic and G.H. Tzeng. Multicriteria planning of postearthquake sustainable reconstruction. Computer-Aided Civil and Infrastructure Engineering, 17(3)(August):211-220, 2002.

[6] Ziping Chiang. Developing an online finanical decision support module based on fuzzy mcdm method and open source tools. In 2009 International Conference on Information and Financial Engineering. IEEE, 2009.

[7] L. Duckstein and S. Opricovic. Multiobjective optimization in river basin development. Water Resources Research, 16(1):1420, 1980.

[8] J. Ye. Fuzzy cross entropy of interval-valued intuitionistic fuzzy sets and its optimal decision-making method based on the weights of alternatives. Expert Systems with Applications, 38:6179-6183, 2011.

[9] Yejun $\mathrm{Xu}$ and Huimin Wang. The induced generalized aggregation operators for intuitionistic fuzzy sets and their application in group decision making. Applied Soft Computing, 12:1168C1179, 2012. 